Pacific

Journal of

Mathematics

\title{
ON HURWITZ SPACES OF COVERINGS WITH ONE SPECIAL FIBER
}

FRANCESCA VETRO 


\title{
ON HURWITZ SPACES OF COVERINGS WITH ONE SPECIAL FIBER
}

\author{
FRANCESCA VETRO
}

\begin{abstract}
Let $X \stackrel{\pi}{\rightarrow} X^{\prime} \stackrel{f}{\rightarrow} Y$ be a covering of smooth, projective complex curves such that $\pi$ is a degree 2 étale covering and $f$ is a degree $d$ covering, with monodromy group $S_{d}$, branched in $n+1$ points one of which is a special point whose local monodromy has cycle type given by the partition $\underline{e}=\left(e_{1}, \ldots, e_{r}\right)$ of $d$. We study such coverings whose monodromy group is either $W\left(B_{d}\right)$ or $w N\left(W\left(B_{d}\right)\right)\left(G_{1}\right) w^{-1}$ for some $w \in W\left(B_{d}\right)$, where $W\left(B_{d}\right)$ is the Weyl group of type $B_{d}, G_{1}$ is the subgroup of $W\left(B_{d}\right)$ generated by reflections with respect to the long roots $\varepsilon_{i}-\varepsilon_{j}$ and $N\left(W\left(B_{d}\right)\right)\left(G_{1}\right)$ is the normalizer of $G_{1}$. We prove that in both cases the corresponding Hurwitz spaces are not connected and hence are not irreducible. In fact, we show that if $n+|\underline{e}| \geq 2 d$, where $|\underline{e}|=\sum_{i=1}^{r}\left(e_{i}-1\right)$, they have $2^{2 g}-1$ connected components.
\end{abstract}

\section{Introduction}

In this paper, we study Hurwitz spaces that parametrize coverings of curves with one special fiber and with monodromy group contained in a Weyl group of type $B_{d}$. We investigate the irreducibility of these spaces and determine their connected components. Coverings whose monodromy group is a Weyl group are interesting because, for example, they appear in the study of spectral curves and of PrymTyurin varieties; see [Donagi 1993; Kanev 1989; 1995; Kanev and Lange 2007]. A natural approach to the study of modular varieties parameterizing Abelian varieties might to build Prym maps from Hurwitz spaces of coverings of curves with monodromy group contained in a Weyl group to such modular varieties. The first question one has to answer here is whether the Hurwitz spaces are irreducible.

We list some previous connectedness results for Hurwitz spaces.

Let $Y$ be a smooth, connected, projective complex curve of genus $\geq 0$, and let $H_{d, n}(Y)$ be the Hurwitz space that parameterizes degree $d$ coverings of $Y$ that are simply branched at $n$ points. Using combinatorial calculations of Clebsch and Lüroth, Hurwitz [1891] showed that $H_{d, n}\left(\mathbb{P}^{1}\right)$ is irreducible. The spaces $H_{d, n}^{o}(Y)$,

MSC2000: primary $14 \mathrm{H} 30$; secondary $14 \mathrm{H} 10$.

Keywords: Hurwitz spaces, connected components, special fiber, Weyl groups of type $B_{d}$. 
which parameterize coverings of curves $Y$ of genus $\geq 1$ with full monodromy group $S_{d}$, were studied by Berstein and Edmonds [1984]. They proved the irreducibility of these spaces under the hypothesis $n>d / 2$.

Let $H_{d, n, \underline{e}}(Y)$ be the Hurwitz space that parameterizes degree $d$ coverings of $Y$ that are simply branched at $n$ points and have one special point whose local monodromy has cycle type given by the partition $\underline{e}=\left(e_{1}, \ldots, e_{r}\right)$ of $d$. Natanzon [1993] and Kluitmann [1988] independently proved the irreducibility of the spaces $H_{d, n, \underline{e}}\left(\mathbb{P}^{1}\right)$, while Kanev [2004] and Vetro [2006] proved the irreducibility of the spaces $H_{d, n, e}^{o}(Y)$, which parameterize coverings of curves of genus $\geq 1$ having full monodromy group $S_{d}$. The best estimate is established in [Vetro 2006], where the irreducibility of $H_{d, n, \underline{e}}^{o}(Y)$ is proved under the hypothesis

$$
n+|\underline{e}| \geq 2 d, \quad \text { where }|\underline{e}|=\sum_{i=1}^{r}\left(e_{i}-1\right) .
$$

Coverings whose monodromy group is a Weyl group and the corresponding Hurwitz spaces were studied in [Biggers and Fried 1986; Kanev 2006; Vetro 2007; 2008a; Vetro 2008b]. Biggers and Fried proved the irreducibility of Hurwitz spaces of coverings of $\mathbb{P}^{1}$ whose monodromy group is a Weyl group of type $D_{d}$, which have simple branching in the sense that each local monodromy is a reflection. Kanev generalized the result to Hurwitz spaces parameterizing Galois coverings of $\mathbb{P}^{1}$ whose Galois group is an arbitrary Weyl group. The author studied Hurwitz spaces parameterizing coverings with one special fiber and monodromy group contained in a Weyl group of type $B_{d}$.

In [Vetro 2007; 2008a; Vetro 2008b], the author studied coverings that can be decomposed as $X \stackrel{\pi}{\longrightarrow} X^{\prime} \stackrel{f}{\longrightarrow} Y$, where $\pi$ is a degree 2 covering and $f$ is a degree $d$ covering, with monodromy group $S_{d}$, having $n$ simply branched points and one special point whose local monodromy has cycle type $\underline{e}$.

The case where $\pi: X \rightarrow X^{\prime}$ is branched was studied in [Vetro 2007; 2008a]. When $\pi$ is branched, the local monodromy at a point of simple branching can be both a reflection with respect to a long root and a reflection with respect to a short root. The Hurwitz spaces that parameterize coverings $X \stackrel{\pi}{\longrightarrow} X^{\prime} \stackrel{f}{\longrightarrow} Y$ with $\pi$ branched are irreducible when $Y \simeq \mathbb{P}^{1}$. In the case in which among the local monodromies there are both reflections with respect to long roots and reflections with respect to short roots, the result generalizes to curves of genus $\geq 1$ under condition (1). In the case of one special fiber and all other local monodromies being reflections with respect to long roots, and under condition (1), there are two possibilities for the monodromy group $G$ : either $G=W\left(B_{d}\right)$ or $G=W\left(D_{d}\right)$, where $W\left(B_{d}\right)$ and $W\left(D_{d}\right)$ are the Weyl groups of type $B_{d}$ and $D_{d}$, respectively. When $G=W\left(D_{d}\right)$, the corresponding Hurwitz spaces are irreducible, while when $G=W\left(B_{d}\right)$ the corresponding Hurwitz spaces have $2^{2 g}-1$ connected components, where $g=g(Y)$. 
The case where $\pi: X \rightarrow X^{\prime}$ is unramified was partially studied in [Vetro 2008b]. It is shown in [Kanev and Lange 2007, Proposition 2.7] that when $\pi: X \rightarrow X^{\prime}$ is unramified and the genus of $Y$ is positive, there are three possible monodromy groups: $W\left(D_{d}\right), W\left(B_{d}\right)$, or $w N\left(W\left(B_{d}\right)\right)\left(G_{1}\right) w^{-1}$ for some $w \in W\left(B_{d}\right)$, where $G_{1}$ is the subgroup of $W\left(B_{d}\right)$ generated by reflections with respect to the long roots of type $\varepsilon_{i}-\varepsilon_{j}$ and $N\left(W\left(B_{d}\right)\right)\left(G_{1}\right)$ is the normalizer of $G_{1}$. The case of $W\left(D_{d}\right)$ was studied in [Vetro 2008b]. The author proved the irreducibility of the corresponding Hurwitz spaces when $Y \simeq \mathbb{P}^{1}$ as well as when, under condition (1), $g(Y) \geq 1$.

This paper completes the study of the problem of irreducibility of Hurwitz spaces of coverings with monodromy group contained in $W\left(B_{d}\right)$, with at most one special fiber, and with a large number of branch points. As discussed above, the remaining cases are those of coverings $X \stackrel{\pi}{\longrightarrow} X^{\prime} \stackrel{f}{\longrightarrow} Y$, where $\pi: X \rightarrow X^{\prime}$ is étale and the monodromy group $G$ is either $W\left(B_{d}\right)$ or conjugate to $N\left(W\left(B_{d}\right)\right)\left(G_{1}\right)$. We prove that in both cases the corresponding Hurwitz spaces are not connected and hence are not irreducible. In fact, we prove under condition (1) that they have $2^{2 g}-1$ connected components, where $g=g(Y)$. Moreover we determine these connected components.

Notation. Here the natural action of $S_{d}$ on $\{1, \ldots, d\}$ is on the right, and we denote the action of $t \in S_{d}$ on $i$ by $i^{t}$. We use $H_{d, n, e}^{o}(Y)$ to denote the Hurwitz space that parameterizes the equivalence classes of degree $d$ coverings of $Y$ with monodromy group $S_{d}$ and with branches at $n+1$ points, $n>0$ of which are points of simple branching and one of which is a special point whose local monodromy has cycle type $\underline{e}$.

\section{Preliminaries: Weyl groups of type $B_{d}$ and braid moves}

1.1. In this subsection, we recall some facts on Weyl groups of type $B_{d}$. The references for this material are [Bourbaki 1968] and [Carter 1972]. Let $d$ be an integer. Consider a real vector space $\mathbb{R}^{d}$ and let $\left\{\varepsilon_{1}, \ldots, \varepsilon_{d}\right\}$ be the standard base of $\mathbb{R}^{d}$. Denote by $R$ the root system $\left\{ \pm \varepsilon_{i}, \pm \varepsilon_{i} \pm \varepsilon_{j}: 1 \leq i, j \leq d\right\}$. The Weyl group of type $B_{d}$, denoted $W\left(B_{d}\right)$, is the group generated by the reflections with respect to the short roots $\varepsilon_{i}$ for $i=1, \ldots, d$, and with respect to the long roots $\varepsilon_{i}-\varepsilon_{j}$ for $1 \leq i<j \leq d$. We will usually denote by $W\left(D_{d}\right)$ the subgroup of $W\left(B_{d}\right)$ generated by the reflections with respect to the long roots $\varepsilon_{i}-\varepsilon_{j}$ and $\varepsilon_{i}+\varepsilon_{j}$ for $1 \leq i<j \leq d$; we denote by $G_{1}$ the subgroup generated by the reflections with respect to the long roots $\varepsilon_{i}-\varepsilon_{j}$ for $1 \leq i<j \leq d$. We will write $N\left(W\left(B_{d}\right)\right)\left(G_{1}\right)$ for the normalizer of $G_{1}$ in $W\left(B_{d}\right)$. It is shown in [Kanev and Lange 2007, Lemma $2.5]$ that the normalizer of $G_{1}$ in $W\left(B_{d}\right)$ is equal to $G_{1} \cup G_{1} \cdot\left(s_{\varepsilon_{1}} s_{\varepsilon_{2}} \cdots s_{\varepsilon_{d}}\right)$; 
furthermore, if $d \geq 3$, the only reflections that belong to $N\left(W\left(B_{d}\right)\right)\left(G_{1}\right)$ are the $s_{\varepsilon_{i}-\varepsilon_{j}}$ with $1 \leq i<j \leq d$.

The elements of $W\left(B_{d}\right)$ act on $\left\{ \pm \varepsilon_{1}, \ldots, \pm \varepsilon_{d}\right\}$ by permutation, and thus every element induces a permutation on $\left\{\left\{\varepsilon_{1},-\varepsilon_{1}\right\}, \ldots,\left\{\varepsilon_{d},-\varepsilon_{d}\right\}\right\}$. The reflection $s_{\varepsilon_{i}}$ exchanges $\varepsilon_{i}$ with $-\varepsilon_{i}$, while leaving fixed those $\varepsilon_{h}$ with $h \neq i$. The reflection $s_{\varepsilon_{i}-\varepsilon_{j}}$ exchanges $\varepsilon_{i}$ and $-\varepsilon_{i}$ with $\varepsilon_{j}$ and $-\varepsilon_{j}$, while fixing $\varepsilon_{h}$ for each $h \neq i, j$. The action of $W\left(B_{d}\right)$ on $\left\{ \pm \varepsilon_{1}, \ldots, \pm \varepsilon_{d}\right\}$ allows us to define an injective homomorphism from $W\left(B_{d}\right)$ into $S_{2 d}$ for which $s_{\varepsilon_{i}-\varepsilon_{j}} \mapsto(i j)(-i-j), s_{\varepsilon_{i}} \mapsto(i-i)$ and $s_{\varepsilon_{i}+\varepsilon_{j}}=$ $s_{\varepsilon_{i}} s_{\varepsilon_{j}} s_{\varepsilon_{i}-\varepsilon_{j}} \mapsto(i-j)(-i j)$.

Let $\left(\mathbb{Z}_{2}\right)^{d}$ be the set of the functions from $\{1, \ldots, d\}$ into $\mathbb{Z}_{2}$ equipped with the sum operation. Let $z \in \mathbb{Z}_{2}$. We will use $z_{i_{1} \ldots i_{e}}$ to denote the function of $\left(\mathbb{Z}_{2}\right)^{d}$ defined as

$$
\begin{array}{cc}
z_{i_{1} \ldots i_{e}}\left(i_{h}\right)=z & \text { for each } \mathrm{h}=1, \ldots, \mathrm{e}, \text { and } \\
z_{i_{1} \ldots i_{e}}(j)=\overline{0} & \text { for each } j \notin\left\{i_{1}, \ldots, i_{e}\right\} .
\end{array}
$$

Let $\Phi$ be the homomorphism from $S_{d}$ in $\operatorname{Aut}\left(\left(\mathbb{Z}_{2}\right)^{d}\right)$ that assigns $\Phi(t) \in \operatorname{Aut}\left(\left(\mathbb{Z}_{2}\right)^{d}\right)$ to $t \in S_{d}$, where $\left[\Phi(t) z^{\prime}\right](j):=z^{\prime}\left(j^{t}\right)$ for each $z^{\prime} \in\left(\mathbb{Z}_{2}\right)^{d}$. Let $\left(\mathbb{Z}_{2}\right)^{d} \times^{s} S_{d}$ be the semidirect product of $\left(\mathbb{Z}_{2}\right)^{d}$ and $S_{d}$ through the homomorphism $\Phi$. Given $\left(z^{\prime} ; t_{1}\right),\left(z^{\prime \prime} ; t_{2}\right) \in\left(\mathbb{Z}_{2}\right)^{d} \times^{s} S_{d}$, we let

$$
\left(z^{\prime} ; t_{1}\right)\left(z^{\prime \prime} ; t_{2}\right):=\left(z^{\prime}+\Phi\left(t_{1}\right) z^{\prime \prime} ; t_{1} t_{2}\right) .
$$

One easily checks that the homomorphism $\Psi: W\left(B_{d}\right) \rightarrow\left(\mathbb{Z}_{2}\right)^{d} \times^{s} S_{d}$ for which $s_{\varepsilon_{i}-\varepsilon_{j}} \mapsto(0 ;(i j)), s_{\varepsilon_{i}} \mapsto\left(\overline{1}_{i} ;\right.$ id $)$ and $s_{\varepsilon_{i}+\varepsilon_{j}} \mapsto\left(\overline{1}_{i j} ;(i j)\right)$ is an isomorphism.

Let $(v ; \eta)$ be an element of $W\left(B_{d}\right)$, where $\eta$ is an $e$-cycle of $S_{d}$ and $v$ is a function of $\left(\mathbb{Z}_{2}\right)^{d}$ that sends to $\overline{0}$ all the indexes fixed by $\eta$.

Definition 1. We call such an element $(v ; \eta)$ in $W\left(B_{d}\right)$ a positive e-cycle if $v$ is either zero or a function that sends to $\overline{1}$ an even number of indexes, and call it negative if it is not positive.

Note that two cycles $(v ; \eta)$ and $\left(v^{\prime} ; \eta^{\prime}\right)$ in $W\left(B_{d}\right)$ are disjoint if $\eta$ and $\eta^{\prime}$ are disjoint. Every element $w$ of $W\left(B_{d}\right)$ can be expressed as a product of disjoint positive and negative cycles. The lengths of these disjoint cycles together with their signs determine the signed cycle type of $w$. It is well known that two elements of $W\left(B_{d}\right)$ are conjugate if and only if they have the same signed cycle type.

Let $\underline{e}=\left(e_{1}, \ldots, e_{r}\right)$ be a partition of $d$ in which $e_{1} \geq \cdots \geq e_{r} \geq 1$. From now on, we will denote by $C_{\underline{e}}$ the conjugate class of $\left(\mathbb{Z}_{2}\right)^{d} \times{ }^{s} S_{d}$ containing elements that are the product of $r$ disjoint positive cycles whose lengths are given by the elements of the partition $\underline{e}$. When $\underline{e}=(2,1, \ldots, 1)$, we will write $C$ for $C_{\underline{e}}$.

1.2. From now on we will denote by $n$ a positive integer. Let $H$ be a group and let $\lambda_{k}, \mu_{k}$, with $k=1, \ldots, g$, be elements of $H$. We will write $[\lambda, \boldsymbol{\mu}]$ for the product $\left[\lambda_{1}, \mu_{1}\right] \cdots\left[\lambda_{g}, \mu_{g}\right]$ of commutators. 
Definition 2. An Hurwitz system with values in $H$ is a $(2 g+n)$-tuple $\left(t_{1}, \ldots, t_{n} ; \lambda_{1}, \mu_{1}, \ldots, \lambda_{g}, \mu_{g}\right)=(\boldsymbol{t} ; \boldsymbol{\lambda}, \boldsymbol{\mu}) \in H^{2 g+n} \quad$ satisfying $t_{1} \cdots t_{n}=[\lambda, \boldsymbol{\mu}]$.

Its entries generate the monodromy group of the system. For $h \in H$, we declare that $(\boldsymbol{t} ; \boldsymbol{\lambda}, \boldsymbol{\mu})$ and $h(\boldsymbol{t} ; \boldsymbol{\lambda}, \boldsymbol{\mu}) h^{-1}$ belong to the same equivalence class, which we denote by $[\boldsymbol{t} ; \boldsymbol{\lambda}, \boldsymbol{\mu}]$.

Note that an $n$-tuple $\left(t_{1}, \ldots, t_{n}\right)=\boldsymbol{t}$ is a Hurwitz system with values in $H$ if $t_{1} \cdots t_{n}=\mathrm{id}$. We denote the equivalence class of $\boldsymbol{t}$ by $[\boldsymbol{t}]$.

We complete this section by recalling some notions on braid moves. The braid groups of a smooth, projective complex curve $Y$ of genus $g \geq 1$ were studied by Birman [1969], Fadell [1962], and Scott [1970]. Let $Y^{(n)}$ be the $n$-fold symmetric product of $Y$, and let $\Delta$ be the codimension 1 locus of $Y^{(n)}$ consisting of nonsimple divisors. The generators of the braid group $\pi_{1}\left(Y^{(n)}-\Delta, D\right)$ are the elementary braids $\sigma_{j}$ with $j=1, \ldots, n-1$ and the braids $\rho_{i k}$ and $\tau_{i k}$ with $1 \leq i \leq n$ and $1 \leq k \leq g$. The generators of $\pi_{1}\left(Y^{(n)}-\Delta, D\right)$ act on Hurwitz systems. The action of the elementary braids $\sigma_{j}$ on Hurwitz systems was studied by Hurwitz [1891], and this was extended to braids $\rho_{i k}$ and $\tau_{i k}$ by Graber, Harris and Starr [2002] and by Kanev [2004]. Here we use results of Hurwitz and Kanev. We associate some generators to a pair of braid moves:

$$
\sigma_{j} \text { to } \sigma_{j}^{\prime}, \sigma_{j}^{\prime \prime}=\left(\sigma_{j}^{\prime}\right)^{-1} ; \quad \rho_{i k} \text { to } \rho_{i k}^{\prime}, \rho_{i k}^{\prime \prime}=\left(\rho_{i k}^{\prime}\right)^{-1} ; \quad \tau_{i k} \text { to } \tau_{i k}^{\prime}, \tau_{i k}^{\prime \prime}=\left(\tau_{i k}^{\prime}\right)^{-1} \text {. }
$$

We call the moves $\sigma_{j}^{\prime}$ and $\sigma_{j}^{\prime \prime}$ elementary moves.

The moves $\sigma_{j}^{\prime}$ and $\sigma_{j}^{\prime \prime}$ fix all the $\lambda_{s}$, all the $\mu_{s}$, and all the $t_{h}$ with $h \neq j, j+1$. They transform $\left(t_{j}, t_{j+1}\right)$ to $\left(t_{j} t_{j+1} t_{j}^{-1}, t_{j}\right)$ and $\left(t_{j+1}, t_{j+1}^{-1} t_{j} t_{j+1}\right)$, respectively.

The braid moves $\rho_{i k}^{\prime}$ and $\rho_{i k}^{\prime \prime}$ fix all the $\lambda_{s}$, all the $t_{h}$ with $h \neq i$ and all the $\mu_{s}$ with $s \neq k$. They modify $t_{i}$ and $\mu_{k}$. Analogously the braid moves $\tau_{i k}^{\prime}$ and $\tau_{i k}^{\prime \prime}$ modify $t_{i}$ and $\lambda_{k}$, leaving unchanged all the $\mu_{s}$, all the $\lambda_{s}$ with $s \neq k$, and all the $t_{h}$ with $h \neq i$. Moreover both $\rho_{i k}^{\prime}$ and $\rho_{i k}^{\prime \prime}$ replace $\mu_{k}$ by an element of type $w \cdot \mu_{k}$, and both $\tau_{i k}^{\prime}$ and $\tau_{i k}^{\prime \prime}$ replace $\lambda_{k}$ by an element of the form $w^{\prime} \cdot \lambda_{k}$, where $w$ and $w^{\prime}$ are elements belonging to the same conjugate class of $t_{i}$. In particular, when

$$
\lambda_{1}=\cdots=\lambda_{k}=\mu_{1}=\cdots=\mu_{k-1}=\mathrm{id},
$$

the braid move $\rho_{1 k}^{\prime}$ transforms $\mu_{k}$ to $t_{1}^{-1} \cdot \mu_{k}$. Analogously when

$$
\lambda_{1}=\cdots=\lambda_{k-1}=\mu_{1}=\cdots=\mu_{k-1}=\mathrm{id},
$$

the move $\tau_{1 k}^{\prime \prime}$ replaces $\lambda_{k}$ by $t_{1}^{-1} \cdot \lambda_{k}$; see [Kanev 2004, Theorem 1.8].

Definition 3. We call two Hurwitz systems with values in $S_{h}$ braid equivalent if one can be obtained from the other using a finite sequence of braid moves $\sigma_{j}^{\prime}, \rho_{i k}^{\prime}, \tau_{i k}^{\prime}$ and their inverses. 


\section{The Hurwitz spaces $H_{W\left(B_{d}\right), n, \underline{e}}(Y)$ and $H_{N\left(W\left(B_{d}\right)\right)\left(G_{1}\right), n, \underline{e}}(Y)$}

2.1. Suppose $X, X^{\prime}$ and $Y$ are smooth, connected, projective complex curves. We assume throughout what follows that $Y$ is a curve of genus $g \geq 1$ and $d$ is an integer greater or equal to 3 . Let $\underline{e}=\left(e_{1}, \ldots, e_{r}\right)$ be a partition of $d$ where $e_{1} \geq \cdots \geq e_{r} \geq 1$. In this paper we study coverings that can be decomposed as $X \stackrel{\pi}{\longrightarrow} X^{\prime} \stackrel{f}{\longrightarrow} Y$, where $\pi$ and $f$ satisfy the following:

$(\star) \pi$ is a degree 2 étale covering and $f$ is a degree $d$ covering, with monodromy group $S_{d}$, branched at $n+1$ points, $n$ of which are points of simple branching and one of which is a special point whose local monodromy has cycle type $\underline{e}$.

Kanev and Lange show in [2007, Proposition 2.7] that the monodromy group of a covering of this type is either $W\left(D_{d}\right)$, or $W\left(B_{d}\right)$ or $w N\left(W\left(B_{d}\right)\right)\left(G_{1}\right) w^{-1}$ for some $w \in W\left(B_{d}\right)$. Coverings $X \stackrel{\pi}{\longrightarrow} X^{\prime} \stackrel{f}{\longrightarrow} Y$ satisfying the conditions $(\star)$ and with monodromy group $W\left(D_{d}\right)$ were studied in [Vetro 2008b]. Here we work with coverings satisfying the conditions $(\star)$ whose monodromy group is either $W\left(B_{d}\right)$ or $w N\left(W\left(B_{d}\right)\right)\left(G_{1}\right) w^{-1}$ for some $w \in W\left(B_{d}\right)$.

Definition 4. Two coverings

$$
X_{1} \stackrel{\pi_{1}}{\longrightarrow} X_{1}^{\prime} \stackrel{f_{1}}{\longrightarrow} Y \quad \text { and } \quad X_{2} \stackrel{\pi_{2}}{\longrightarrow} X_{2}^{\prime} \stackrel{f_{2}}{\longrightarrow} Y
$$

are said to be equivalent if there exist two biholomorphic maps $p: X_{1} \rightarrow X_{2}$ and $p^{\prime}: X_{1}^{\prime} \rightarrow X_{2}^{\prime}$ such that $p^{\prime} \circ \pi_{1}=\pi_{2} \circ p$ and $f_{2} \circ p^{\prime}=f_{1}$. The equivalence class containing the covering $X \stackrel{\pi}{\longrightarrow} X^{\prime} \stackrel{f}{\longrightarrow} Y$ is denoted by $\left[X \stackrel{\pi}{\longrightarrow} X^{\prime} \stackrel{f}{\longrightarrow} Y\right]$.

We write $H_{G, n, \underline{e}}(Y)$ for the Hurwitz space that parameterizes equivalence classes of coverings $X \stackrel{\pi}{\longrightarrow} X^{\prime} \stackrel{f}{\longrightarrow} Y$ satisfying the conditions $(\star)$ whose monodromy group is conjugated to $G$, where $G$ is either $W\left(B_{d}\right)$ or $N\left(W\left(B_{d}\right)\right)\left(G_{1}\right)$.

From now on we will denote by $D$ and by $m: \pi_{1}\left(Y-D, b_{0}\right) \rightarrow S_{2 d}$ respectively the branch locus and the monodromy homomorphism associated to the covering $X \stackrel{\pi}{\longrightarrow} X^{\prime} \stackrel{f}{\longrightarrow} Y$. The image via $m$ of a standard generating system for $\pi_{1}\left(Y-D, b_{0}\right)$ determines an equivalence class $[\boldsymbol{t} ; \boldsymbol{\lambda}, \boldsymbol{\mu}]$ of Hurwitz systems with values in $\left(\mathbb{Z}_{2}\right)^{d} \times{ }^{s} S_{d} \simeq W\left(B_{d}\right)$ and monodromy group conjugated to $G$ such that $n$ among the $t_{j}$ belong to $C$ and one belongs to $C_{\underline{e}}$. We denote by $A_{G, n, \underline{e}, g}$ the set of all equivalence classes of Hurwitz systems as above. Let $\delta: H_{G, n, \underline{e}}(Y) \rightarrow Y^{(n+1)}-\Delta$ be the map that assigns to each equivalence class $\left[X \stackrel{\pi}{\longrightarrow} X^{\prime} \stackrel{f}{\longrightarrow} Y\right]$ the branch locus $D$ of $X \stackrel{\pi}{\longrightarrow} X^{\prime} \stackrel{f}{\longrightarrow} Y$. By Riemann's existence theorem we can identify the fiber of $\delta$ over $D$ with $A_{G, n, \underline{e}, g}$. There is a unique topology on $H_{G, n, \underline{e}}(Y)$ such that $\delta$ is a topological covering map; see [Fulton 1969]. Therefore the braid group $\pi_{1}\left(Y^{(n+1)}-\Delta, D\right)$ acts on $A_{G, n, \underline{e}, g}$. The orbits of this action are in one-toone correspondence with the connected components of $H_{G, n, \underline{e}}(Y)$. So in order to 
determine the connected components of $H_{G, n, \underline{e}}(Y)$, it is enough to find the orbits of the action of $\pi_{1}\left(Y^{(n+1)}-\Delta, D\right)$ on $A_{G, n, \underline{e}, g}$.

2.2. From now on we associate to the partition $\underline{e}=\left(e_{1}, \ldots, e_{r}\right)$ of $d$ in which $e_{1} \geq \cdots \geq e_{r} \geq 1$ the element

$$
\left(12 \ldots e_{1}\right)\left(e_{1}+1 \ldots e_{1}+e_{2}\right) \cdots\left(\left(e_{1}+\cdots+e_{r-1}\right)+1 \ldots d\right) \in S_{d} .
$$

We will also denote the permutation (2) by

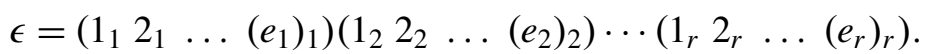

We write $q_{i}$ for the cycle $\left(1_{i} 2_{i} \ldots\left(e_{i}\right)_{i}\right)$ and $|\underline{e}|$ for $\sum_{i=1}^{r}\left(e_{i}-1\right)$. We use $\widetilde{Z}_{i}$ with $i=1, \ldots, r$ to denote the sequence $\left(\left(0 ;\left(1_{i} 2_{i}\right)\right),\left(0 ;\left(1_{i} 3_{i}\right)\right), \ldots,\left(0 ;\left(1_{i}\left(e_{i}\right)_{i}\right)\right)\right)$ and $\widetilde{Z}$ to denote the concatenation $\widetilde{Z}_{1} \widetilde{Z}_{2} \ldots \widetilde{Z}_{r}$.

Lemma 1 [Kanev 2004, Main Lemma 2.1]. Let $(\boldsymbol{t} ; \boldsymbol{\lambda}, \boldsymbol{\mu})$ be a Hurwitz system with values in $\left(\mathbb{Z}_{2}\right)^{d} \times{ }^{s} S_{d}$. Suppose that $t_{i} t_{i+1}=(0 ;$ id $)$. Let $H$ be the subgroup of $\left(\mathbb{Z}_{2}\right)^{d} \times^{s} S_{d}$ generated by $\left\{t_{1}, \ldots, t_{i-1}, t_{i+2}, \ldots, t_{n}, \lambda_{1}, \mu_{1}, \ldots, \lambda_{g}, \mu_{g}\right\}$. Then for every $h \in H$, the given Hurwitz system is braid equivalent to

$$
\left(t_{1}, \ldots, t_{i-1}, h^{-1} t_{i} h, h^{-1} t_{i+1} h, t_{i+2}, \ldots, t_{n} ; \boldsymbol{\lambda}, \boldsymbol{\mu}\right) .
$$

Proposition 1 [Vetro 2008b, Proposition 3]. Let $[\boldsymbol{t}$ ] be an equivalence class of Hurwitz systems with values in $\left(\mathbb{Z}_{2}\right)^{d} \times^{s} S_{d} \simeq W\left(B_{d}\right)$ such that $n$ among the $t_{j}$ belong to $C$ and one belongs to $C_{\underline{e}}$ and such that if $t_{j}=\left(z^{\prime} ; t_{j}^{\prime}\right)$ for $j=1, \ldots, n+1$, the group generated by the permutations $t_{j}^{\prime}$ is all of $S_{d}$.

(i) If $r>1$, then $[t]$ is braid equivalent to a class of the form

$$
\begin{aligned}
{\left[\boldsymbol{t}_{1}\right]=\left[\widetilde{Z},\left(0 ;\left(1_{1} 1_{2}\right)\right),\left(0 ;\left(1_{1} 1_{2}\right)\right)\right.} & \ldots,\left(0 ;\left(1_{1} 1_{r-1}\right)\right),\left(0 ;\left(1_{1} 1_{r-1}\right)\right), \\
& \left.\left(z_{1_{1} 1_{r}}^{1} ;\left(1_{1} 1_{r}\right)\right), \ldots,\left(z_{1_{1} 1_{r}}^{s} ;\left(1_{1} 1_{r}\right)\right),\left(0 ; \epsilon^{-1}\right)\right],
\end{aligned}
$$

where each $\left(0 ;\left(1_{1} 1_{i}\right)\right)$ with $2 \leq i \leq r-1$ appears twice, the $z^{h}$ are elements of $\mathbb{Z}_{2}$, and $s$ is an even positive integer.

(ii) If $r=1$, then $[\boldsymbol{t}]$ is braid equivalent to a class of the form

$$
\left[\boldsymbol{t}_{2}\right]=\left[\widetilde{Z}_{1},\left(z_{1_{1} 2_{1}}^{1} ;\left(1_{1} 2_{1}\right)\right), \ldots,\left(z_{1_{1} 2_{1}}^{s} ;\left(1_{1} 2_{1}\right)\right),\left(0 ; \epsilon^{-1}\right)\right],
$$

where the $z^{h}$ are elements of $\mathbb{Z}_{2}$ and $s$ is an even positive integer.

Observation 1. Note that if in $\boldsymbol{t}_{1}$ there are elements of type $\left(\overline{1}_{1_{1} 1_{r}} ;\left(1_{1} 1_{r}\right)\right)$, then they are even in number. This follows from the relation

$$
\begin{aligned}
&\left(0 ;\left(1_{1} 2_{1}\right)\right) \cdots\left(0 ;\left(1_{1}\left(e_{1}\right)_{1}\right)\right) \cdots\left(0 ;\left(1_{r} 2_{r}\right)\right) \cdots\left(0 ;\left(1_{r}\left(e_{r}\right)_{r}\right)\right) \\
& \cdot\left(0 ;\left(1_{1} 1_{2}\right)\right) \cdots\left(0 ;\left(1_{1} 1_{r-1}\right)\right)\left(z_{1_{1} 1_{r}}^{1} ;\left(1_{1} 1_{r}\right)\right) \cdots\left(z_{1_{1} 1_{r}}^{s} ;\left(1_{1} 1_{r}\right)\right)=(0 ; \epsilon) .
\end{aligned}
$$


Analogously one deduces that if in $\boldsymbol{t}_{2}$ there are elements of type $\left(\overline{1}_{1_{1} 2_{1}} ;\left(1_{1} 2_{1}\right)\right)$, they are even in number.

\section{The connected components of $H_{W\left(B_{d}\right), n, \underline{e}}(Y)$}

In this section we study the spaces $H_{W\left(B_{d}\right), n, \underline{e}}(Y)$. We show that these spaces are not connected and hence they are not irreducible. We prove, in fact, that the Hurwitz spaces $H_{W\left(B_{d}\right), n, \underline{e}}(Y)$ have $2^{2 g}-1$ connected components for $n+|\underline{e}| \geq 2 d$.

Proposition 2. Suppose condition (1) holds.

(i) If $r>1$, each equivalence class $[\boldsymbol{t} ; \boldsymbol{\lambda}, \boldsymbol{\mu}]$ in $A_{W\left(B_{d}\right), n, \underline{e}, \mathrm{~g}}$ is braid equivalent to a class of the form $\left[T_{1} ; \boldsymbol{c}, \boldsymbol{d}\right]$, where $T_{1}$ is the sequence

$\left(\widetilde{Z},\left(0 ;\left(1_{1} 1_{2}\right)\right),\left(0 ;\left(1_{1} 1_{2}\right)\right), \ldots,\left(0 ;\left(1_{1} 1_{r-1}\right)\right),\left(0 ;\left(1_{1} 1_{r-1}\right)\right)\right.$,

$$
\left.\left(0 ;\left(1_{1} 1_{r}\right)\right), \ldots,\left(0 ;\left(1_{1} 1_{r}\right)\right),\left(0 ; \epsilon^{-1}\right)\right)
$$

in which each $\left(0 ;\left(1_{1} 1_{i}\right)\right)$ for $2 \leq i \leq r-1$ appears twice, while $\left(0 ;\left(1_{1} 1_{r}\right)\right)$ appears an even number of times.

(ii) If $r=1$ each equivalence class $[\boldsymbol{t} ; \boldsymbol{\lambda}, \boldsymbol{\mu}]$ in $A_{W\left(B_{d}\right), n, \underline{e}, g}$ is braid equivalent to a class of the form $\left[T_{2} ; \boldsymbol{c}, \boldsymbol{d}\right]$, where $T_{2}$ is the sequence

$$
\left(\widetilde{Z}_{1},\left(0 ;\left(1_{1} 2_{1}\right)\right), \ldots,\left(0 ;\left(1_{1} 2_{1}\right)\right),\left(0 ; \epsilon^{-1}\right)\right)
$$

in which $\left(0 ;\left(1_{1} 2_{1}\right)\right)$ appears an even number of times.

Moreover $(\boldsymbol{c}, \boldsymbol{d})$ is the sequence $\left(\left(c_{1} ; \mathrm{id}\right),\left(d_{1} ; \mathrm{id}\right), \ldots,\left(c_{g} ; \mathrm{id}\right),\left(d_{g} ; \mathrm{id}\right)\right)$, where the functions $c_{k}$ and $d_{k}$ for $k=1, \ldots, g$ are equal to either 0 or $\overline{1}_{1}$ and at least one among the $c_{k}$ or the $d_{k}$ is different from 0.

Proof. Step 1. At first we prove that every class $[\boldsymbol{t} ; \boldsymbol{\lambda}, \boldsymbol{\mu}]$ in $A_{W\left(B_{d}\right), n, \underline{e}, g}$ is braid equivalent to a class of the form $\left[\hat{t}_{1}, \ldots, \hat{t}_{n},\left(* ; \epsilon^{-1}\right) ; \boldsymbol{c}, \boldsymbol{d}\right]$. Let $t_{j}=\left(* ; t_{j}^{\prime}\right)$, $\lambda_{k}=\left(* ; \lambda_{k}^{\prime}\right)$ and $\mu_{k}=\left(* ; \mu_{k}^{\prime}\right)$ for $j=1, \ldots, n+1$ and $k=1, \ldots, g$. By Riemann's existence theorem, the equivalence class of Hurwitz systems $\left[\boldsymbol{t}^{\prime} ; \lambda^{\prime}, \boldsymbol{\mu}^{\prime}\right]$ corresponds to an equivalence class of coverings belonging to $H_{d, n, e}^{o}(Y)$. By (1), the Hurwitz space $H_{d, n, e}^{o}(Y)$ is irreducible; see [Vetro 2006, Theorem 1]. Therefore $\left[\boldsymbol{t}^{\prime} ; \lambda^{\prime}, \boldsymbol{\mu}^{\prime}\right]$ is braid equivalent to a class of the form $\left[t_{1}^{\prime \prime}, \ldots, t_{n}^{\prime \prime}, \epsilon^{-1} ; \mathrm{id}, \ldots, \mathrm{id}\right]$, and so $[\boldsymbol{t} ; \boldsymbol{\lambda}, \boldsymbol{\mu}]$ is braid equivalent to $\left[\tilde{t}_{1}, \ldots, \tilde{t}_{n},\left(* ; \epsilon^{-1}\right) ; \boldsymbol{a}, \boldsymbol{b}\right]$, where $(\boldsymbol{a}, \boldsymbol{b})$ is the sequence $\left(\left(a_{1} ; \mathrm{id}\right),\left(b_{1} ; \mathrm{id}\right), \ldots,\left(a_{g} ; \mathrm{id}\right),\left(b_{g} ; \mathrm{id}\right)\right)$.

If $a_{k}$ and $b_{k}$ for $k=1, \ldots, g$ are equal to either $\overline{1}_{1}$ or 0 , we obtain the claim. So we suppose that $a_{1}$ is different from $\overline{1}_{1}$ and 0 .

Note that if $a_{1}=\overline{1}_{i}$ for $i \in\{2, \ldots, d\}$ and if among the $\tilde{t}_{h}$ there are both $\left(\overline{1}_{1 i} ;(1 i)\right)$ and $(0 ;(1 i))$, then our class is braid equivalent to a class of the form $\left[\ldots,\left(* ; \epsilon^{-1}\right) ;\left(\overline{1}_{1} ; \mathrm{id}\right),\left(b_{1} ; \mathrm{id}\right), \ldots,\left(a_{g} ; \mathrm{id}\right),\left(b_{g} ; \mathrm{id}\right)\right]$. In fact, using elementary moves $\sigma_{l}^{\prime \prime}$ we can move to the first place $(0 ;(1 i))$, and we then apply the $\tau_{11}^{\prime \prime}$ 
that transforms $\left(a_{1} ;\right.$ id $)$ to $(0 ;(1 i))\left(a_{1} ;\right.$ id $)$. After that, we move to the first place $\left(\overline{1}_{1 i} ;(1 i)\right)$ and again act by $\tau_{11}^{\prime \prime}$. By this process, we can replace $\left(a_{1} ;\right.$ id $)$ with $\left(\overline{1}_{1 i} ; \mathrm{id}\right)\left(\overline{1}_{i} ; \mathrm{id}\right)=\left(\overline{1}_{1} ; \mathrm{id}\right)$. Analogously we check that if $i$ and $j$ are two indexes sent to $\overline{1}$ by $a_{1}$ and if in our Hurwitz system among the $\tilde{t}_{h}$ there are both $\left(\overline{1}_{i j} ;(i j)\right)$ and $(0 ;(i j))$, then our class is braid equivalent to a class of the form $\left[\ldots,\left(* ; \epsilon^{-1}\right) ;\left(\hat{a}_{1} ; \mathrm{id}\right),\left(b_{1} ; \mathrm{id}\right), \ldots,\left(a_{g} ; \mathrm{id}\right),\left(b_{g} ; \mathrm{id}\right)\right]$, where $\hat{a}_{1}$ is a function that sends to $\overline{1}$ the same indexes sent to $\overline{1}$ by $a_{1}$ except $i$ and $j$.

Let $i, j$ be indexes belonging to $\{1, \ldots, d\}$. From what we have just said it follows that, if acting by elementary moves $\sigma_{h}^{\prime}, \sigma_{h}^{\prime \prime}$ with $1 \leq h \leq n$, we are able to replace a class of type $\left[\tilde{t}_{1}, \ldots, \tilde{t}_{n},\left(* ; \epsilon^{-1}\right) ; \boldsymbol{a}, \boldsymbol{b}\right]$ belonging to $A_{W\left(B_{d}\right), n, e, g}$ with one of the form $\left[\ldots,\left(\overline{1}_{i j} ;(i j)\right), \ldots,(0 ;(i j)), \ldots,\left(* ; \epsilon^{-1}\right) ; \boldsymbol{a}, \boldsymbol{b}\right]$, then after a finite number of steps we can obtain a class braid equivalent to ours of type either

$$
\begin{aligned}
& {\left[\ldots,\left(* ; \epsilon^{-1}\right) ;(0 ; \mathrm{id}),\left(b_{1} ; \mathrm{id}\right), \ldots,\left(a_{g} ; \mathrm{id}\right),\left(b_{g} ; \mathrm{id}\right)\right] \text { or }} \\
& {\left[\ldots,\left(* ; \epsilon^{-1}\right) ;\left(\overline{1}_{1} ; \mathrm{id}\right),\left(b_{1} ; \mathrm{id}\right), \ldots,\left(a_{g} ; \mathrm{id}\right),\left(b_{g} ; \mathrm{id}\right)\right],}
\end{aligned}
$$

depending on whether $a_{1}$ sends to $\overline{1}$ an even or odd number of indexes.

So far we proved that our class is braid equivalent to $\left[\tilde{t}_{1}, \ldots, \tilde{t}_{n},\left(* ; \epsilon^{-1}\right) ; \boldsymbol{a}, \boldsymbol{b}\right]$. The relation $[\boldsymbol{a}, \boldsymbol{b}]=(0$; id $)$ implies that $\tilde{t}_{1} \cdots \tilde{t}_{n}\left(* ; \epsilon^{-1}\right)=(0$; id $)$. Since the group generated by the permutations corresponding to the $\tilde{t}_{j}$ is $S_{d}$, the equivalence class of Hurwitz systems $\left[\tilde{t}_{1}, \ldots, \tilde{t}_{n},\left(* ; \epsilon^{-1}\right)\right]$ satisfies the hypothesis of Proposition 1 and thus it is braid equivalent to a class of the form $\left[\boldsymbol{t}_{1}\right]$ or $\left[\boldsymbol{t}_{2}\right]$, depending on whether $r>1$ or $r=1$. Note that to replace the class $\left[\tilde{t}_{1}, \ldots, \tilde{t}_{n},\left(* ; \epsilon^{-1}\right)\right]$ with one of the form $\left[\boldsymbol{t}_{1}\right]$ or $\left[\boldsymbol{t}_{2}\right]$, we use elementary moves $\sigma_{j}^{\prime}$ and $\sigma_{j}^{\prime \prime}$, and we conjugate the elements in our Hurwitz system with elements of type $\left(\overline{1}_{i \ldots h}\right.$; id); see [Vetro $2008 \mathrm{~b}$, proof of Proposition 3]. Since $\left(\overline{1}_{i \ldots h} ;\right.$ id $)\left(a_{k} ;\right.$ id $)\left(\overline{1}_{i \ldots h} ;\right.$ id $)=\left(a_{k} ;\right.$ id $)$ and $\left(\overline{1}_{i \ldots h} ; \mathrm{id}\right)\left(b_{k} ; \mathrm{id}\right)\left(\overline{1}_{i \ldots h} ; \mathrm{id}\right)=\left(b_{k} ; \mathrm{id}\right)$, we can conclude by Proposition 1 that $\left[\tilde{t}_{1}, \ldots, \tilde{t}_{n},\left(* ; \epsilon^{-1}\right) ; \boldsymbol{a}, \boldsymbol{b}\right]$ is braid equivalent to a class of the form $\left[\boldsymbol{t}_{1} ; \boldsymbol{a}, \boldsymbol{b}\right]$ or $\left[\boldsymbol{t}_{2} ; \boldsymbol{a}, \boldsymbol{b}\right]$ depending on whether $r>1$ or $r=1$. Previously we proved under the hypothesis (1) that every Hurwitz system of type $\left(\boldsymbol{t}_{i} ; \boldsymbol{a}, \boldsymbol{b}\right)$ is braid equivalent to a Hurwitz system of the form $\left(\ldots,\left(\overline{1}_{i j} ;(i j)\right), \ldots,(0 ;(i j)), \ldots,\left(0 ; \epsilon^{-1}\right) ; \boldsymbol{a}, \boldsymbol{b}\right)$; see [Vetro 2008b, Theorem 2, Step 2]. Because of this we can confirm that, acting by braid moves, it is possible to transform $\left[\tilde{t}_{1}, \ldots, \tilde{t}_{n},\left(* ; \epsilon^{-1}\right) ; \boldsymbol{a}, \boldsymbol{b}\right]$ to

$$
\left[\ldots,\left(* ; \epsilon^{-1}\right) ;\left(c_{1} ; \mathrm{id}\right),\left(b_{1} ; \mathrm{id}\right), \ldots,\left(a_{g} ; \mathrm{id}\right),\left(b_{g} ; \mathrm{id}\right)\right] .
$$

Once we prove that our class is braid equivalent to a class of the form $\left[\hat{\boldsymbol{t}} ;\left(c_{1} ; \mathrm{id}\right),\left(b_{1} ; \mathrm{id}\right), \ldots,\left(a_{g} ; \mathrm{id}\right),\left(b_{g} ; \mathrm{id}\right)\right]$, we replace $\left(b_{1} ;\right.$ id $)$ with $\left(d_{1} ;\right.$ id $)$ by proceeding in the same way but using the braid move $\rho_{11}^{\prime}$. We reason analogously when $a_{k}$ is different from 0 and $\overline{1}_{1}$ and $a_{l}$ and $b_{l}$ are equal to 0 or $\overline{1}_{1}$ for each $l \leq k-1$, but we use instead the braid moves $\tau_{1 k}^{\prime \prime}$. In the end, if $b_{k}$ is different from 
0 and $\overline{1}_{1}$ and $a_{l}, b_{l}, a_{k}$ are equal to 0 or $\overline{1}_{1}$ for each $l \leq k-1$, we replace $\left(b_{k}\right.$; id) with $\left(d_{k} ;\right.$ id) by applying the braid moves $\rho_{1 k}^{\prime}$. So we obtain the claim.

Step 2. In Step 1 we proved that $[\boldsymbol{t} ; \boldsymbol{\lambda}, \boldsymbol{\mu}]$ is braid equivalent to a class of the form $\left[\hat{t}_{1}, \ldots, \hat{t}_{n},\left(* ; \epsilon^{-1}\right) ; \boldsymbol{c}, \boldsymbol{d}\right]$. From the relation $[\boldsymbol{c}, \boldsymbol{d}]=(0 ;$ id $)$, we deduce that $\hat{t}_{1} \cdots \hat{t}_{n}=(* ; \epsilon)$ and thus $(* ; \epsilon) \in\left\langle\hat{t}_{1}, \ldots, \hat{t}_{n}\right\rangle$. Since the monodromy group of the Hurwitz systems belonging to our class is $W\left(B_{d}\right)$ and the $\hat{t}_{h}$ belong to $C$, at least one among the $c_{k}$ or the $d_{k}$ is equal to $\overline{1}_{1}$.

From the relation $[\boldsymbol{c}, \boldsymbol{d}]=(0$; id $)$, we also deduce that $\left[\hat{t}_{1}, \ldots, \hat{t}_{n},\left(* ; \epsilon^{-1}\right)\right]$ satisfies all the hypothesis of Proposition 1 ; because of this $\left[\hat{t}_{1}, \ldots, \hat{t}_{n},\left(* ; \epsilon^{-1}\right) ; \boldsymbol{c}, \boldsymbol{d}\right]$ is braid equivalent to a class of type $\left[\boldsymbol{t}_{1} ; \boldsymbol{c}, \boldsymbol{d}\right]$ or $\left[\boldsymbol{t}_{2} ; \boldsymbol{c}, \boldsymbol{d}\right]$ depending on whether $r>1$ or $r=1$. If $r>1$ and the elements of type $\left(* ;\left(1_{1} 1_{r}\right)\right)$ in $t_{1}$ are all equal to $\left(0 ;\left(1_{1} 1_{r}\right)\right)$ or if $r=1$ and the element of type $\left(* ;\left(1_{1} 2_{1}\right)\right)$ in $\boldsymbol{t}_{2}$ are all equal to $\left(0 ;\left(1_{1} 2_{1}\right)\right)$, we have the normal form required. Otherwise using elementary moves, depending on whether $r>1$ or $r=1$, we place nearby the elements of type $\left(\overline{1}_{1_{1} 1_{r}} ;\left(1_{1} 1_{r}\right)\right)$ that are in $\boldsymbol{t}_{1}$ or the elements of type $\left(\overline{1}_{1_{1} 2_{1}} ;\left(1_{1} 2_{1}\right)\right)$ that are in $\boldsymbol{t}_{2}$. Observe that condition (1) insures that in $\boldsymbol{t}_{1}$ there are at least four elements of type $\left(* ;\left(1_{1} 1_{r}\right)\right)$ and in $\boldsymbol{t}_{2}$ there are at least three elements of type $\left(* ;\left(1_{1} 2_{1}\right)\right)$. Since at least one among the $c_{k}$ or the $d_{k}$ is equal to $\overline{1}_{1}$, if we cancel two among the $\left(* ;\left(1_{1} 1_{r}\right)\right)$ in $\boldsymbol{t}_{1}$ or two among the $\left(* ;\left(1_{1} 2_{1}\right)\right)$ in $\boldsymbol{t}_{2}$, the group generated by the remaining elements of the Hurwitz systems $\left(\boldsymbol{t}_{j} ; \boldsymbol{c}, \boldsymbol{d}\right)$ is still $W\left(B_{d}\right)$. Because of this, using Lemma 1, we can replace if $r>1$ every pair

$$
\left(\left(\overline{1}_{1_{1} 1_{r}} ;\left(1_{1} 1_{r}\right)\right),\left(\overline{1}_{1_{1} 1_{r}} ;\left(1_{1} 1_{r}\right)\right)\right) \quad \text { of } \boldsymbol{t}_{1} \text { with }\left(\left(0 ;\left(1_{1} 1_{r}\right)\right),\left(0 ;\left(1_{1} 1_{r}\right)\right)\right)
$$

and if $r=1$ every pair

$$
\left(\left(\overline{1}_{1_{1} 2_{1}} ;\left(1_{1} 2_{1}\right)\right),\left(\overline{1}_{1_{1} 2_{1}} ;\left(1_{1} 2_{1}\right)\right)\right) \text { of } \boldsymbol{t}_{2} \text { with }\left(\left(0 ;\left(1_{1} 2_{1}\right)\right),\left(0 ;\left(1_{1} 2_{1}\right)\right)\right) \text {; }
$$

it sufficient to choose $h=\left(\overline{1}_{1_{1}} ;\right.$ id). Since number of the $\left(\overline{1}_{1_{1} 1_{r}} ;\left(1_{1} 1_{r}\right)\right)$ in $\boldsymbol{t}_{1}$ and the number of $\left(\overline{1}_{1_{1} 2_{1}} ;\left(1_{1} 2_{1}\right)\right)$ in $\boldsymbol{t}_{2}$ are both even (see Observation 1$)$, the proposition is proved.

Let $\left\{h_{1}, \ldots, h_{s}\right\}$ and $\left\{k_{1}, \ldots, k_{l}\right\}$ be two subsets of $\{1, \ldots, g\}$ such that at least one among $s$ and $l$ is greater of 0 . Let us denote by $\left[\hat{T}_{1}\right]_{\left\{h_{1}, \ldots, h_{s}\right\},\left\{k_{1}, \ldots, k_{l}\right\}}$ and $\left[\hat{T}_{2}\right]_{\left\{h_{1}, \ldots, h_{s}\right\},\left\{k_{1}, \ldots, k_{l}\right\}}$ the equivalence classes $\left[T_{1} ; \boldsymbol{c}, \boldsymbol{d}\right]$ and $\left[T_{2} ; \boldsymbol{c}, \boldsymbol{d}\right]$, respectively, where $T_{1}, T_{2}$ and $(\boldsymbol{c}, \boldsymbol{d})$ are the sequences defined in (i) and (ii) of Proposition 2 and where the $c_{h}$ and the $d_{k}$ with $h \in\left\{h_{1}, \ldots, h_{s}\right\}$ and $k \in\left\{k_{1}, \ldots, k_{l}\right\}$ are equal to $\overline{1}_{1}$, while all others are equal to 0 .

Note that the equivalence class $\left[\hat{T}_{i}\right]_{\left\{h_{1}, \ldots, h_{s}\right\},\left\{k_{1}, \ldots, k_{l}\right\}}$ contains only Hurwitz systems of type

$$
\left(t ;\left(a_{1} ; \mathrm{id}\right),\left(b_{1} ; \mathrm{id}\right), \ldots,\left(a_{g} ; \mathrm{id}\right),\left(b_{g} ; \mathrm{id}\right)\right)
$$


where the $a_{h}$ and the $b_{k}$ with $h \in\left\{h_{1}, \ldots, h_{s}\right\}$ and $k \in\left\{k_{1}, \ldots, k_{l}\right\}$ are equal to $\overline{1}_{i}$ for some $i \in\{1, \ldots, d\}$, while all others are equal to 0 . In fact, if we conjugate ( 0 ; id) and $\left(\overline{1}_{1} ;\right.$ id $)$ with $(a ; s) \in\left(\mathbb{Z}_{2}\right)^{d} \times^{s} S_{d}$, we obtain $(a ; s)(0 ;$ id $)(a ; s)^{-1}=(0 ;$ id $)$, while

$(a ; s)\left(\overline{1}_{1} ; \mathrm{id}\right)(a ; s)^{-1}=\left(a+\Phi(s) \overline{1}_{1} ; s\right)(a ; s)^{-1}=\left(\overline{1}_{i} ;\right.$ id $) \quad$ with $i \in\{1, \ldots, d\}$.

Because of this, we can confirm that there are $2^{2 g}-1$ equivalence classes of the form $\left[\hat{T}_{1}\right]_{\left\{h_{1}, \ldots, h_{s}\right\},\left\{k_{1}, \ldots, k_{l}\right\}}$, and another $2^{2 g}-1$ of the form $\left[\hat{T}_{2}\right]_{\left\{h_{1}, \ldots, h_{s}\right\},\left\{k_{1}, \ldots, k_{l}\right\}}$.

Theorem 1. Suppose (1) holds. The connected components of $H_{W\left(B_{d}\right), n, \underline{e}}(Y)$ are of number $2^{2 g}-1$ and are in one-to-one correspondence with the orbits of the equivalence classes $\left[\hat{T}_{1}\right]_{\left\{h_{1}, \ldots, h_{s}\right\},\left\{k_{1}, \ldots, k_{l}\right\}}$ or $\left[\hat{T}_{2}\right]_{\left\{h_{1}, \ldots, h_{s}\right\},\left\{k_{1}, \ldots, k_{l}\right\}}$, depending on whether $r>1$ or $r=1$.

Proof. The connected components of $H_{W\left(B_{d}\right), n, \underline{e}}(Y)$ are in one-to-one correspondence with the orbits of the action of $\pi_{1}\left(Y^{(n+1)}-\Delta, D\right)$ on $A_{W\left(B_{d}\right), n, e, g}$. Since (1) holds, by Proposition 2 every equivalence class belonging to $A_{W\left(B_{d}\right), n, \underline{e}, g}$ is braid equivalent to a class of the form $\left[\hat{T}_{1}\right]_{\left\{h_{1}, \ldots, h_{s}\right\},\left\{k_{1}, \ldots, k_{l}\right\}}$ or $\left[\hat{T}_{2}\right]_{\left\{h_{1}, \ldots, h_{s}\right\},\left\{k_{1}, \ldots, k_{l}\right\}}$, depending on whether $r>1$ or $r=1$. It follows that the connected components of $H_{W\left(B_{d}\right), n, \underline{e}}(Y)$ are in bijection with the orbits of the equivalence classes $\left[\hat{T}_{1}\right]_{\left\{h_{1}, \ldots, h_{s}\right\},\left\{k_{1}, \ldots, k_{l}\right\}}$ or $\left[\hat{T}_{2}\right]_{\left\{h_{1}, \ldots, h_{s}\right\},\left\{k_{1}, \ldots, k_{l}\right\}}$, depending on whether $r>1$ or $r=1$.

The equivalence classes $\left[\hat{T}_{i}\right]_{\left\{h_{1}, \ldots, h_{s}\right\},\left\{k_{1}, \ldots, k_{l}\right\}}$ belong to different orbits of the action of $\pi_{1}\left(Y^{(n+1)}-\Delta, D\right)$ on $A_{W\left(B_{d}\right), n, \underline{e}, g}$. In fact, the only braid moves that change $\left(c_{k}\right.$; id) are $\tau_{i k}^{\prime}, \tau_{i k}^{\prime \prime}$ and sequences of braid moves of this type. The moves $\tau_{i k}^{\prime}$ and $\tau_{i k}^{\prime \prime}$ act on $\left(c_{k} ; \mathrm{id}\right)$ transforming it to $w^{\prime} \cdot\left(c_{k} ; \mathrm{id}\right)$, where $w^{\prime}$ is an element belonging either to $C$ or to $C_{\underline{e}}$ (see Section 1.2). Therefore $w^{\prime}$ is an element of form $(a ; \xi)$, where $a$ is either zero or a function that sends an even number of indexes to $\overline{1}$. From this we deduce that, by acting by sequences of braid moves $\tau_{i k}^{\prime}, \tau_{j k}^{\prime \prime}$ on an element $\left(c_{k} ;\right.$ id) of the form ( 0 ; id), we can replace $(0 ;$ id) with an element of form $(a ; \xi)$, but not with an element of form $(b ; \xi)$, where $b$ is a function that sends an odd number of indexes to $\overline{1}$. Note that we arrive at the same conclusion if we reason on the $\left(d_{k} ;\right.$ id $)$ and on the moves $\rho_{i k}^{\prime}, \rho_{i k}^{\prime \prime}$.

Since the classes of the form $\left[\hat{T}_{i}\right]_{\left\{h_{1}, \ldots, h_{s}\right\},\left\{k_{1}, \ldots, k_{l}\right\}}$ are $2^{2 g}-1$, the Hurwitz space $H_{W\left(B_{d}\right), n, \underline{e}}(Y)$ has $2^{2 g}-1$ connected components.

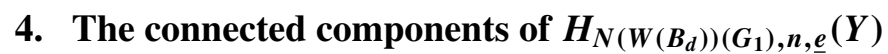

In this section we fix our attention on the Hurwitz spaces $H_{N\left(W\left(B_{d}\right)\right)\left(G_{1}\right), n, \underline{e}}(Y)$. We prove under condition (1) that they have $2^{2 g}-1$ connected components, and furthermore we determine these connected components.

From now on we will write $\overline{1}_{12 \ldots d}$ for the function of $\left(\mathbb{Z}_{2}\right)^{d}$ that sends to $\overline{1}$ each index $h \in\{1, \ldots, d\}$. 
Proposition 3. Under condition (1), each equivalence class $[t ; \lambda, \mu]$ belonging to $A_{N}\left(W\left(B_{d}\right)\right)\left(G_{1}\right), n, \underline{e}, g$ is braid equivalent to a class of the form

(i) $\left[T_{1} ; \boldsymbol{l}, \boldsymbol{m}\right]$ if $r>1$, or

(ii) $\left[T_{2} ; \boldsymbol{l}, \boldsymbol{m}\right]$ if $r=1$,

where $(\boldsymbol{l}, \boldsymbol{m})$ is the sequence

$$
\left(\left(l_{1} ; \mathrm{id}\right),\left(m_{1} ; \mathrm{id}\right), \ldots,\left(l_{g} ; \mathrm{id}\right),\left(m_{g} ; \mathrm{id}\right)\right)
$$

and where the functions $l_{k}$ and $m_{k}$ are equal either to 0 or to $\overline{1}_{12 \ldots d}$ and at least one among the $l_{k}$ or the $m_{k}$ is different from 0. Moreover, $T_{1}$ and $T_{2}$ are the sequences defined respectively in (i) and (ii) of Proposition 2.

Proof. Let $t_{j}=\left(* ; t_{j}^{\prime}\right), \lambda_{k}=\left(* ; \lambda_{k}^{\prime}\right)$ and $\mu_{k}=\left(* ; \mu_{k}^{\prime}\right)$ for $j=1, \ldots, n+1$ and $k=1, \ldots, g$. By Riemann's existence theorem, the equivalence class of Hurwitz systems $\left[\boldsymbol{t}^{\prime} ; \lambda^{\prime}, \boldsymbol{\mu}^{\prime}\right]$ corresponds to an equivalence class of coverings belonging to $H_{d, n, \underline{e}}^{o}(Y)$. By condition (1), the Hurwitz space $H_{d, n, \underline{e}}^{o}(Y)$ is irreducible (see [Vetro 2006, Theorem 1]) and therefore it is possible, acting by braid moves, to replace $\left[\boldsymbol{t}^{\prime} ; \lambda^{\prime}, \boldsymbol{\mu}^{\prime}\right]$ with a class of the form $\left[t_{1}^{\prime \prime}, \ldots, t_{n}^{\prime \prime}, \epsilon^{-1} ;\right.$ id $\left., \ldots, \mathrm{id}\right]$. It follows that $[\boldsymbol{t} ; \boldsymbol{\lambda}, \boldsymbol{\mu}]$ is braid equivalent to a class of type $\left[\tilde{t}_{1}, \ldots, \tilde{t}_{n},\left(* ; \epsilon^{-1}\right) ; \boldsymbol{a}, \boldsymbol{b}\right]$, where $(\boldsymbol{a}, \boldsymbol{b})$ is the sequence $\left(\left(a_{1} ; \mathrm{id}\right),\left(b_{1} ; \mathrm{id}\right), \ldots,\left(a_{g} ; \mathrm{id}\right),\left(b_{g} ; \mathrm{id}\right)\right)$.

The relation $[\boldsymbol{a}, \boldsymbol{b}]=(0$; id $)$ implies that $\tilde{t}_{1} \cdots \tilde{t}_{n}\left(* ; \epsilon^{-1}\right)=(0$; id $)$. Since the group generated by the transpositions corresponding to the $\tilde{t}_{j}$ is $S_{d}$, we can confirm that the equivalence class $\left[\tilde{t}_{1}, \ldots, \tilde{t}_{n},\left(* ; \epsilon^{-1}\right)\right]$ satisfies all the hypothesis of Proposition 1 , and thus it is braid equivalent to a class of the form $\left[\boldsymbol{t}_{1}\right]$ or $\left[\boldsymbol{t}_{2}\right]$, depending on whether $r>1$ or $r=1$. Because of this we can replace the sequence $\left(\tilde{t}_{1}, \ldots, \tilde{t}_{n},\left(* ; \epsilon^{-1}\right)\right)$ by $\boldsymbol{t}_{1}$ or $\boldsymbol{t}_{2}$ depending on whether $r>1$ or $r=1$, obtaining that our class is braid equivalent to a class of the form $\left[\boldsymbol{t}_{i} ; \boldsymbol{a}, \boldsymbol{b}\right]$ (recall that in proving Proposition 2, we already observed that in replacing $\left(\tilde{t}_{1}, \ldots, \tilde{t}_{n},\left(* ; \epsilon^{-1}\right)\right)$ with $\boldsymbol{t}_{1}$ or $\boldsymbol{t}_{2}$, we leave unchanged the elements of type $\left(a_{k} ; \mathrm{id}\right)$ and $\left.\left(b_{k} ; \mathrm{id}\right)\right)$.

Now we separately discuss the cases $r>1$ and $r=1$.

Case: $r>1$. The Hurwitz systems belonging to the class $\left[\boldsymbol{t}_{1} ; \boldsymbol{a}, \boldsymbol{b}\right]$ have monodromy group conjugate to $N\left(W\left(B_{d}\right)\right)\left(G_{1}\right)$; this insures that in $\boldsymbol{t}_{1}$ among the elements of type $\left(z_{1_{1} 1_{r}}^{h} ;\left(1_{1} 1_{r}\right)\right)$ for $h=1, \ldots, s$, there are not both $\left(\overline{1}_{1_{1} 1_{r}} ;\left(1_{1} 1_{r}\right)\right)$ and $\left(0 ;\left(1_{1} 1_{r}\right)\right)$. In fact, the group $H$ generated by the elements of the sequence $t_{1}$ contains, for each $h \in\{1, \ldots, d\} \backslash\left\{1_{1}\right\}$ and for each $k \in\{1, \ldots, d\} \backslash\left\{1_{r}\right\}$ with $h \neq k$, an element of the type $\left(* ;\left(1_{1} h\right)\right)$ and one of type $\left(* ;\left(1_{r} k\right)\right)$. If $\left(\overline{1}_{1_{1} 1_{r}} ;\left(1_{1} 1_{r}\right)\right)$ and $\left(0 ;\left(1_{1} 1_{r}\right)\right)$ are both in $t_{1}$, then by conjugating $\left(* ;\left(1_{1} h\right)\right)$ with $\left(\overline{1}_{1_{1} 1_{r}} ;\left(1_{1} 1_{r}\right)\right)$ and $\left(0 ;\left(1_{1} 1_{r}\right)\right)$, we obtain that both $\left(\overline{1}_{h 1_{r}} ;\left(h 1_{r}\right)\right)$ and $\left(0 ;\left(h 1_{r}\right)\right)$ belong to $H$. By conjugating $\left(\overline{1}_{h 1_{r}} ;\left(h 1_{r}\right)\right)$ and $\left(0 ;\left(h 1_{r}\right)\right)$ with $\left(* ;\left(1_{r} k\right)\right)$, we obtain that both $\left(\overline{1}_{h k} ;(h k)\right)$ and $(0 ;(h k))$ belong to $H$. Since $h$ and $k$ are arbitrary indexes in 
$\{1, \ldots, d\}$, this implies that $H=W\left(D_{d}\right)$. So the monodromy group of the Hurwitz system $\left(\boldsymbol{t}_{1} ; \boldsymbol{a}, \boldsymbol{b}\right)$ is either $W\left(D_{d}\right)$ or $W\left(B_{d}\right)$, a contradiction.

Now we observe that if the $\left(z_{1_{1} 1_{r}}^{h} ;\left(1_{1} 1_{r}\right)\right)$ in $\boldsymbol{t}_{1}$ are of the form $\left(\overline{1}_{1_{1} 1_{r}} ;\left(1_{1} 1_{r}\right)\right)$, we can replace the system $\left(t_{1} ; \boldsymbol{a}, \boldsymbol{b}\right)$ by the system $\left(T_{1} ; \boldsymbol{a}, \boldsymbol{b}\right)$ by conjugating any element in $\left(\boldsymbol{t}_{1} ; \boldsymbol{a}, \boldsymbol{b}\right)$ with $\left(\overline{1}_{1_{r} 2_{r} \ldots\left(e_{r}\right)_{r}} ;\right.$ id), where $\overline{1}_{1_{r} 2_{r} \ldots\left(e_{r}\right)_{r}}$ is the function that sends to $\overline{1}$ only the indexes moved by $q_{r}$. In fact, in our system in addition to the elements $\left(\overline{1}_{1_{1} 1_{r}} ;\left(1_{1} 1_{r}\right)\right),\left(0 ; \epsilon^{-1}\right),\left(a_{k} ;\right.$ id $)$ and $\left(b_{k} ;\right.$ id $)$, there are elements of type $\left(z_{\alpha \beta} ;(\alpha \beta)\right)$, where the indexes $\alpha$ and $\beta$ are moved both either by $q_{r}$ or by a cycle different from $q_{r}$. One easily checks that elements of form $\left(z_{\alpha \beta} ;(\alpha \beta)\right)$ are unchanged when conjugated by $\left(\overline{1}_{1_{r} \ldots\left(e_{r}\right)_{r}} ;\right.$ id $)$. Analogously, the elements $\left(0 ; \epsilon^{-1}\right)$, $\left(a_{k} ;\right.$ id $)$ and $\left(b_{k} ;\right.$ id $)$ are unchanged under conjugation by $\left(\overline{1}_{1_{r}} 2_{r} \ldots\left(e_{r}\right)_{r} ;\right.$ id $)$, while $\left(\overline{1}_{1_{1} 1_{r}} ;\left(1_{1} 1_{r}\right)\right)$ conjugated by $\left(\overline{1}_{1_{r} 2_{r} \ldots\left(e_{r}\right)_{r}} ;\right.$ id) becomes $\left(0 ;\left(1_{1} 1_{r}\right)\right)$.

Since the monodromy group $G$ of the Hurwitz system $\left(T_{1} ; \boldsymbol{a}, \boldsymbol{b}\right)$ is conjugated to $N\left(W\left(B_{d}\right)\right)\left(G_{1}\right)$, we are sure that at least one among the $a_{k}$ or the $b_{k}$ is different from 0 . Furthermore, we can confirm that the $a_{k}$ and the $b_{k}$ that are different from 0 are equal to $\overline{1}_{12 \ldots d}$. In fact, we suppose by way of contradiction that $a_{k}$ for some $k$ is different from 0 and $\overline{1}_{12 \ldots d}$. Then $a_{k}$ sends to $\overline{0}$ an index $i$ and to $\overline{1}$ an index $j$. Since $(0 ;(i j))$ belongs to the group generated by the elements in $T_{1}$ and since conjugating $(0 ;(i j))$ by $\left(a_{k} ;\right.$ id) gives $\left(\overline{1}_{i j} ;(i j)\right)$, we deduce that both $\left(\overline{1}_{i j} ;(i j)\right)$ and $(0 ;(i j))$ belong to $G$. This implies that $G$ contains, for each $h, k \in\{1, \ldots, d\}$, both $\left(\overline{1}_{h k} ;(h k)\right)$ and $(0 ;(h k))$. Thus $G$ is either $W\left(D_{d}\right)$ or $W\left(B_{d}\right)$, a contradiction. Note that if we suppose that $b_{k}$ is different from 0 and $\overline{1}_{12 \ldots d}$ for some $k$, we arrive at the same contradiction. This complete the proof in the case $r>1$.

Case: $r=1$. Because the Hurwitz systems belonging to the class $\left[\boldsymbol{t}_{2} ; \boldsymbol{a}, \boldsymbol{b}\right]$ have monodromy group $G$ conjugated to $N\left(W\left(B_{d}\right)\right)\left(G_{1}\right)$, cannot there be in $\boldsymbol{t}_{2}$ both $\left(\overline{1}_{1_{1} 2_{1}} ;\left(1_{1} 2_{1}\right)\right)$ and $\left(0 ;\left(1_{1} 2_{1}\right)\right)$ (see the case $\left.r>1\right)$. So the elements of type $\left(z_{1_{1} 2_{1}}^{h} ;\left(1_{1} 2_{1}\right)\right)$ in $\boldsymbol{t}_{2}$ must all be equal to $\left(0 ;\left(1_{1} 2_{1}\right)\right)$, that is, the sequence $\boldsymbol{t}_{2}$ is of the form $T_{2}$. Moreover, since $G$ is conjugated to $N\left(W\left(B_{d}\right)\right)\left(G_{1}\right)$, the $a_{k}$ and $b_{k}$ cannot be all equal to 0 . Since the functions $a_{k}$ and $b_{k}$ cannot be different from 0 and from $\overline{1}_{12 \ldots d}$ (see the case $r>1$ ), the proposition is proved.

Observation 2. Note that the equivalence classes defined in Proposition 3 (i), (ii) are not braid equivalent to ones defined in Proposition 2 (i), (ii). In fact, the former have monodromy group conjugate to $N\left(W\left(B_{d}\right)\right)\left(G_{1}\right)$ while the latter have as monodromy group all of $W\left(B_{d}\right)$.

Let $\left\{h_{1}, \ldots, h_{s}\right\}$ and $\left\{k_{1}, \ldots, k_{v}\right\}$ be two subsets of $\{1, \ldots, g\}$ such that at least one among $s$ and $v$ is greater than 0 . Let us denote by $\left[\tilde{T}_{1}\right]_{\left\{h_{1}, \ldots, h_{s}\right\},\left\{k_{1}, \ldots, k_{v}\right\}}$ and $\left[\tilde{T}_{2}\right]_{\left\{h_{1}, \ldots, h_{s}\right\},\left\{k_{1}, \ldots, k_{v}\right\}}$ the equivalence classes of the Hurwitz systems

$$
\left(\tilde{T}_{1}\right)_{\left\{h_{1}, \ldots, h_{s}\right\},\left\{k_{1}, \ldots, k_{v}\right\}}=\left(T_{1} ; \boldsymbol{l}, \boldsymbol{m}\right) \quad \text { and } \quad\left(\tilde{T}_{2}\right)_{\left\{h_{1}, \ldots, h_{s}\right\},\left\{k_{1}, \ldots, k_{v}\right\}}=\left(T_{2} ; \boldsymbol{l}, \boldsymbol{m}\right),
$$


respectively, where $T_{1}, T_{2}$ and $(\boldsymbol{l}, \boldsymbol{m})$ are the sequences defined in (i) and (ii) of Proposition 2 and where the $l_{h}$ and the $m_{k}$ with $h \in\left\{h_{1}, \ldots, h_{s}\right\}$ and $k \in\left\{k_{1}, \ldots, k_{v}\right\}$ are equal to $\overline{1}_{12 \ldots d}$, while all others equal 0 .

If we conjugate $\left(0 ;\right.$ id) and $\left(\overline{1}_{12 \ldots d}\right.$; id) with an element $(a ; s) \in\left(\mathbb{Z}_{2}\right)^{d} \times{ }^{s} S_{d}$, we leave them unchanged. Therefore the class $\left[\tilde{T}_{i}\right]_{\left\{h_{1}, \ldots, h_{s}\right\},\left\{k_{1}, \ldots, k_{v}\right\}}$ contains only Hurwitz systems of type $(\boldsymbol{t} ; \boldsymbol{l}, \boldsymbol{m})$, where the $l_{h}$ and the $m_{k}$ with $h \in\left\{h_{1}, \ldots, h_{s}\right\}$ and $k \in\left\{k_{1}, \ldots, k_{v}\right\}$ are equal to $\overline{1}_{12 \ldots d}$, while all others are 0 . From this we deduce that there are $2^{2 g}-1$ equivalence classes of the form $\left[\tilde{T}_{1}\right]_{\left\{h_{1}, \ldots, h_{s}\right\},\left\{k_{1}, \ldots, k_{v}\right\}}$ and another $2^{2 g}-1$ of the form $\left[\tilde{T}_{2}\right]_{\left\{h_{1}, \ldots, h_{s}\right\},\left\{k_{1}, \ldots, k_{v}\right\}}$.

Theorem 2. Suppose (1) holds. The number of the connected components of $H_{N\left(W\left(B_{d}\right)\right)\left(G_{1}\right), n, \underline{e}}(Y)$ is $2^{2 g}-1$. The connected components of $H_{N\left(W\left(B_{d}\right)\right)\left(G_{1}\right), n, \underline{e}}(Y)$ are in one-to-one correspondence with the orbits of the equivalence classes $\left[\tilde{T}_{1}\right]_{\left\{h_{1}, \ldots, h_{s}\right\},\left\{k_{1}, \ldots, k_{v}\right\}}$ or $\left[\tilde{T}_{2}\right]_{\left\{h_{1}, \ldots, h_{s}\right\},\left\{k_{1}, \ldots, k_{v}\right\}}$, depending on whether $r>1$ or $r=1$.

Proof. Since the connected components of $H_{N\left(W\left(B_{d}\right)\right)\left(G_{1}\right), n, \underline{e}}(Y)$ are in bijection with the orbits of the action of $\pi_{1}\left(Y^{(n+1)}-\Delta, D\right)$ on $A_{N}\left(W\left(B_{d}\right)\right)\left(G_{1}\right), n, \underline{e}, g$ and by (1), Proposition 3 gives that the connected components of $H_{N\left(W\left(B_{d}\right)\right)\left(G_{1}\right), n, \underline{e}}(Y)$ are in bijection with the orbits of the equivalence classes $\left[\tilde{T}_{1}\right]_{\left\{h_{1}, \ldots, h_{s}\right\},\left\{k_{1}, \ldots, k_{v}\right\}}$ or $\left[\tilde{T}_{2}\right]_{\left\{h_{1}, \ldots, h_{s}\right\},\left\{k_{1}, \ldots, k_{v}\right\}}$, depending on whether $r>1$ or $r=1$.

Hurwitz systems of the form $\left(\tilde{T}_{i}\right)_{\left\{h_{1}, \ldots, h_{s}\right\},\left\{k_{1}, \ldots, k_{v}\right\}}$ are not braid equivalent. In fact, acting by the braid moves $\rho_{j k}^{\prime}, \rho_{j k}^{\prime \prime}, \tau_{j h}^{\prime}, \tau_{j h}^{\prime \prime}$, we can replace $t_{j}=\left(0 ; t_{j}^{\prime}\right)$ only with an element belonging to the same conjugate class of form $(0 ; \xi)$ (see [Kanev 2004, Theorem 1.8]). Hence acting with the moves $\tau_{j k}^{\prime}$ and $\tau_{j k}^{\prime \prime}$ we can replace $\left(l_{k} ;\right.$ id) with $(0 ; \xi) \cdot\left(l_{k} ;\right.$ id $)$. So, acting by sequences of braid moves $\tau_{i k}^{\prime}$ and $\tau_{j k}^{\prime \prime}$, we can replace one $\left(l_{k} ;\right.$ id) of the form $\left(0 ;\right.$ id) with $(0 ; \xi)$ and one $\left(l_{k} ;\right.$ id) of the form $\left(\overline{1}_{12 \ldots d} ;\right.$ id) with $\left(\overline{1}_{12 \ldots d} ; \xi\right)$, but we cannot transform $(0 ;$ id $)$ to $\left(\overline{1}_{12 \ldots d} ;\right.$ id $)$. Using that reasoning with the $\left(m_{k}\right.$; id) and with the braid moves $\rho_{i k}^{\prime}$ and $\rho_{i k}^{\prime \prime}$, one arrives at the same conclusion, and therefore the equivalence classes $\left[\tilde{T}_{i}\right]_{\left\{h_{1}, \ldots, h_{s}\right\},\left\{k_{1}, \ldots, k_{v}\right\}}$ belong to different orbits of the action of $\pi_{1}\left(Y^{(n+1)}-\Delta, D\right)$ on $A_{N\left(W\left(B_{d}\right)\right)\left(G_{1}\right), n, e, g}$.

Since the classes of the form $\left[\tilde{T}_{i}\right]_{\left\{h_{1}, \ldots, h_{s}\right\},\left\{k_{1}, \ldots, k_{v}\right\}}$ are $2^{2 g}-1$, the Hurwitz space $H_{N\left(W\left(B_{d}\right)\right)\left(G_{1}\right), n, \underline{e}}(Y)$ has $2^{2 g}-1$ connected components.

\section{Acknowledgment}

I thank the referee for your very useful suggestions and remarks.

\section{References}

[Berstein and Edmonds 1984] I. Berstein and A. L. Edmonds, "On the classification of generic branched coverings of surfaces”, Illinois Journal of Math. 28:1 (1984), 64-82. MR 85k:57004 Zbl 0551.57001 
[Biggers and Fried 1986] R. Biggers and M. Fried, "Irreducibility of moduli spaces of cyclic unramified covers of genus $g$ curves", Trans. Amer. Math. Soc. 295:1 (1986), 59-70. MR 87f:14011 Zbl 0601.14022

[Birman 1969] J. S. Birman, “On braid groups”, Comm. Pure Appl. Math. 22 (1969), 41-72. MR 38 \#2764 Zbl 0157.30904

[Bourbaki 1968] N. Bourbaki, Éléments de mathématique, Fasc. XXXIV: Groupes et algèbres de Lie, Chapitre IV: Groupes de Coxeter et systèmes de Tits, Chapitre V: Groupes engendrés par des réflexions, Chapitre VI: systèmes de racines, Actualités Scientifiques et Industrielles 1337, Hermann, Paris, 1968. MR 39 \#1590 Zbl 0186.33001

[Carter 1972] R. W. Carter, "Conjugacy classes in the Weyl group", Compositio Math. 25 (1972), 1-59. MR 47 \#6884 Zbl 0254.17005

[Donagi 1993] R. Donagi, "Decomposition of spectral covers", pp. 145-175 in Journées de Géométrie Algébrique d'Orsay (Orsay, 1992), Astérisque 218, Société Mathématique de France, Paris, 1993. MR 95f:14065 Zbl 0820.14031

[Fadell and Neuwirth 1962] E. Fadell and L. Neuwirth, "Configuration spaces", Math. Scand. 10 (1962), 111-118. MR 25 \#4537 Zbl 0136.44104

[Fulton 1969] W. Fulton, "Hurwitz schemes and irreducibility of moduli of algebraic curves", Ann. of Math. (2) 90 (1969), 542-575. MR 41 \#5375 Zbl 0194.21901

[Graber et al. 2002] T. Graber, J. Harris, and J. Starr, "A note on Hurwitz schemes of covers of a positive genus curve”, preprint, 2002. arXiv math.AG/0205056

[Hurwitz 1891] A. Hurwitz, "Ueber Riemann'sche Flächen mit gegebenen Verzweigungspunkten", Math. Ann. 39:1 (1891), 1-60. MR 1510692 JFM 23.0429.01

[Kanev 1989] V. Kanev, "Spectral curves, simple Lie algebras, and Prym-Tjurin varieties", pp. 627645 in Theta functions - Bowdoin 1987, I (Brunswick, ME, 1987), edited by L. Ehrenpreis and R. C. Gunning, Proc. Sympos. Pure Math. 49, Amer. Math. Soc., Providence, RI, 1989. MR 91b: 14028 Zbl 0707.14041

[Kanev 1995] V. Kanev, "Spectral curves and Prym-Tjurin varieties, I", pp. 151-198 in Abelian varieties (Egloffstein, 1993), edited by H. Lange et al., de Gruyter, Berlin, 1995. MR 96d:14024 Zbl 0856.14010

[Kanev 2004] V. Kanev, "Irreducibility of Hurwitz spaces", preprint 241, Dipartimento di Matematica ed Applicazioni, Università degli Studi di Palermo, 2004. arXiv math.AG/0509154

[Kanev 2006] V. Kanev, "Hurwitz spaces of Galois coverings of $\mathbb{P}^{1}$, whose Galois groups are Weyl groups", J. Algebra 305:1 (2006), 442-456. MR 2007g:14032 Zbl 1118.14034

[Kanev and Lange 2007] V. Kanev and H. Lange, "Polarization types of isogenous Prym-Tyurin varieties", preprint 332, Dipartimento di Matematica ed Applicazioni, Università degli Studi di Palermo, 2007. arXiv 0707.0364v1

[Kluitmann 1988] P. Kluitmann, "Hurwitz action and finite quotients of braid groups", pp. 299-325 in Braids (Santa Cruz, CA, 1986), edited by J. S. Birman and A. Libgober, Contemp. Math. 78, Amer. Math. Soc., Providence, RI, 1988. MR 90d:20071 Zbl 0701.20019

[Natanzon 1993] S. M. Natanzon, "Topology of 2-dimensional coverings and meromorphic functions on real and complex algebraic curves", Selecta Mathematica Sovietica 12:3 (1993), 251-291. MR 95f:57005

[Scott 1970] G. P. Scott, "Braid groups and the group of homeomorphisms of a surface", Proc. Cambridge Philos. Soc. 68 (1970), 605-617. MR 42 \#3786 Zbl 0203.56302

[Vetro 2006] F. Vetro, "Irreducibility of Hurwitz spaces of coverings with one special fiber", Indag. Math. (N.S.) 17:1 (2006), 115-127. MR 2008j:14054 Zbl 1101.14040 
[Vetro 2007] F. Vetro, "Irreducibility of Hurwitz spaces of coverings with monodromy groups Weyl groups of type $W\left(B_{d}\right)$ ", Boll. Unione Mat. Ital. Sez. B Artic. Ric. Mat. (8) 10:2 (2007), 405-431. MR 2008f:14043 Zbl 05230819

[Vetro 2008a] F. Vetro, "Connected components of Hurwitz spaces of coverings with one special fiber and monodromy groups contained in a Weyl group of type $B_{d}$ ", Boll. Unione Mat. Ital. (9) 1:1 (2008), 87-103. MR 2387999 Zbl 05320540

[Vetro 2008b] F. Vetro, "Irreducibility of Hurwitz spaces of coverings with one special fiber and monodromy group a Weyl group of type $D_{d}$ ", Manuscripta Math. 125:3 (2008), 353-368. MR 2008j:14055 Zbl 1139.14023

Received December 13, 2007. Revised November 13, 2008.

FRANCESCA VETRO

Dipartimento di Matematica ed APPliCAZIONI

UNIVERSITÀ DEGLI STUdi DI PALERMO

VIA ARCHIRAFI 34

90123 PALERMO

ITALY

fvetro@math.unipa.it 